New Advances on Disease Biomarkers and Molecular Targets in Biomedicine 

Nikki P. Lee • C.Y. Cheng • John M. Luk Editors

\section{New Advances on Disease Biomarkers and Molecular Targets in Biomedicine}




\section{Editors}

Nikki P. Lee

Department of Surgery

University of Hong Kong

Hong Kong, China

John M. Luk

Department of Pharmacology

National University of Singapore

Singapore

Department of Surgery

National University of Singapore

Singapore

\author{
C.Y. Cheng \\ The Mary M. Wohlford Laboratory \\ for Male Contraceptive Research \\ Center for Biomedical Research \\ Population Council \\ New York, NY, USA
}

ISBN 978-1-62703-455-5

ISBN 978-1-62703-456-2 (eBook)

DOI 10.1007/978-1-62703-456-2

Springer New York Heidelberg Dordrecht London

Library of Congress Control Number: 2013940517

\section{(C) Springer Science+Business Media New York 2013}

This work is subject to copyright. All rights are reserved by the Publisher, whether the whole or part of the material is concerned, specifically the rights of translation, reprinting, reuse of illustrations, recitation, broadcasting, reproduction on microfilms or in any other physical way, and transmission or information storage and retrieval, electronic adaptation, computer software, or by similar or dissimilar methodology now known or hereafter developed. Exempted from this legal reservation are brief excerpts in connection with reviews or scholarly analysis or material supplied specifically for the purpose of being entered and executed on a computer system, for exclusive use by the purchaser of the work. Duplication of this publication or parts thereof is permitted only under the provisions of the Copyright Law of the Publisher's location, in its current version, and permission for use must always be obtained from Springer. Permissions for use may be obtained through RightsLink at the Copyright Clearance Center. Violations are liable to prosecution under the respective Copyright Law.

The use of general descriptive names, registered names, trademarks, service marks, etc. in this publication does not imply, even in the absence of a specific statement, that such names are exempt from the relevant protective laws and regulations and therefore free for general use.

While the advice and information in this book are believed to be true and accurate at the date of publication, neither the authors nor the editors nor the publisher can accept any legal responsibility for any errors or omissions that may be made. The publisher makes no warranty, express or implied, with respect to the material contained herein.

Printed on acid-free paper

Humana Press is a brand of Springer

Springer is part of Springer Science+Business Media (www.springer.com) 


\section{Preface}

Biomarkers and molecular targets are two indispensable themes in diverse disciplines of research and development in modern medicine. Insights derived from these studies would impact on current therapies or management of human diseases, as well as translation of studies from lab bench to clinical practices for improving health of patients. Advances in research over the past decades have led to a remarkable increase in understanding of disease etiologies/pathological conditions, preventive measures and treatment options, management of diseases, in particularly cancers and unexplained infertility in men, however still remain a major hurdle nowadays. Still much work remains to be done taking the advantage of recent advances in biotechnologies, proteomics, and genomics.

This monograph summarizes the latest research in various aspects of biomarkers and molecular targets ranging from studies in biomolecules, cellular, and/or signaling pathways that regulate disease metabolisms and mechanisms, with a hope to identify leading disease-specific biomarkers and therapeutic targets for predicting diagnosis and prognosis, classifying therapeutic responses, and enhancing treatment efficacy.

We hope that this book can be an introduction for students and interns on the breakthroughs of biomedical research, a reference work for researchers on their topics of interest, a comprehensive guide for clinicians on the updates in the field, and for those with passion in science and research who want to gain an insight in this rapidly developing area. In this expanding era of scientific discovery, the concepts and ideas discussed here will be revised and augmented periodically. It is our goal that an integration of the existing knowledge with new evolving concepts from the literatures in the years to come can promote future progress and bridge the gap between findings in the laboratory and translational medicine.

This book cannot be published without the enthusiasm and dedication of all the contributing authors, who are willing to use their valuable time to share their knowledge and wisdom with our readers through this monograph. More importantly, we 
would like to express our sincere thanks to all the staff at Springer, in particular Mindy Okura-Marszycki, Aleta Kalkstein, and Renata Hutter, for their efforts and dedication in working with us selflessly during the preparation and production of this book.

Hong Kong, China

Nikki P. Lee

New York, NY, USA

C.Y. Cheng

Shanghai, China

John M. Luk 


\section{Contents}

1 Nonreceptor Protein Kinases c-Src, c-Yes, and FAK Are Biomarkers for Male Contraceptive Research

Xiang Xiao, Dolores D. Mruk, Pranitha Jenardhanan, Jayakanthan Mannu, Premendu P. Mathur, Will M. Lee, Bruno Silvestrini, Yan-ho Cheng, and C.Y. Cheng

2 Ankyrin Repeat and Suppressor of Cytokine Signaling Box (ASB) Family Members for Cancer Diagnosis, Prognosis, and Treatment.

Victor Au and Nikki P. Lee

3 MicroRNA as Cancer Biomarkers and Targets

Kwong-Fai Wong, Xiaoou Jiang, and John M. Luk

4 Oncofetal Molecules as Biomarkers and Drug Targets for Hepatic Cancer

Felix H. Shek, Tracy C.W. Lai, Sarwat Fatima, and Nikki P. Lee

5 Ion Channels as Novel Pancreatic Cancer Biomarkers and Targets

Nelson S. Yee and Rosemary K. Yee

6 Circulating Biomarkers for Esophageal Squamous

Cell Carcinoma

Kenneth K. Lai, Kin-Tak Chan, Sarwat Fatima, Nikki P. Lee, and Simon Law

7 Using Genomic Biomarkers to Predict Patient Prognosis and Treatment Response in Gastric Cancer Kakoli Das and Patrick Tan

8 CARF Regulates Cellular Senescence and Apoptosis through p53-Dependent and -Independent Pathways

Renu Wadhwa, Rumani Singh, Zeenia Kaul, and Sunil C. Kaul 
9 Targeting Cancer Metabolisms

Angela M. Liu, Wei Wang, and John M. Luk

10 Modulation of Autophagy as a Novel Cancer

Therapeutic Strategy

Jianzhou Cui and Han-Ming Shen

11 Functional Proteomics Screening for Novel

Anti-viral Drug Targets

Shengtao Zhou, Manchao Kuang, Xia Zhao, and Canhua Huang

12 Identification of Tumor Antigens as Targets

for Novel Antitumor Therapies

Yanyan Han and Qing-Yu He

13 Mesoporous Silica Nanoparticles for Cancer Therapy

Qian Liu and Weiliang Xia

14 Chemoresistance in Glioma.

Stella Sun, Derek Lee, and Gilberto K.K. Leung

About the Editors

Index 


\section{Contributors}

Victor Au Department of Surgery, The University of Hong Kong, Hong Kong, China

Kin-Tak Chan Department of Surgery, The University of Hong Kong, Hong Kong, China

C.Y. Cheng The Mary M. Wohlford Laboratory for Male Contraceptive Research, Center for Biomedical Research, Population Council, New York, NY, USA

Yan-ho Cheng Richmond University Medical Center, Staten Island, NY, USA

Jianzhou Cui Saw Swee Hock School of Public Health, Department of Physiology, Yong Loo Lin School of Medicine, National University of Singapore, Singapore

Kakoli Das Cancer and Stem Cell Biology, Duke-NUS Graduate Medical School, Singapore, Singapore

Sarwat Fatima Department of Surgery, The University of Hong Kong, Hong Kong, China

School of Chinese Medicine, Hong Kong Baptist University, Hong Kong, China

Yanyan Han Key Laboratory of Functional Protein Research of Guangdong Higher Education Institutes, Institute of Life and Health Engineering, Jinan University, Guangzhou, P.R. China

Qing-Yu He Key Laboratory of Functional Protein Research of Guangdong Higher Education Institutes, Institute of Life and Health Engineering, Jinan University, Guangzhou, P.R. China

Department of Biochemistry \& Molecular Biology, College of Life Science and Technology, Jinan University, Guangzhou, P.R. China

Canhua Huang The State Key Laboratory of Biotherapy, West China Hospital, Sichuan University, Chengdu, P.R. China 
Pranitha Jenardhanan Center for Bioinformatics, School of Life Sciences, Pondicherry University, Pondicherry, India

Xiaoou Jiang Department of Pharmacology, National University of Singapore, Singapore

Department of Surgery, National University of Singapore, Singapore, Singapore

Sunil C. Kaul National Institute of Advanced Industrial Science \& Technology (AIST), Tsukuba Science City, Japan

Zeenia Kaul Department of Molecular Virology, Immunology and Medical Genetics, The Ohio State University Wexner Cancer Center, College of Medicine, Columbus, $\mathrm{OH}$, USA

Manchao Kuang Department of Gynecology and Obstetrics, West China Second Hospital, Sichuan University, Chengdu, P.R. China

Kenneth K. Lai Department of Surgery, The University of Hong Kong, Hong Kong, China

Tracy C.W. Lai The Jockey Club School of Public Health and Primary Care, The Chinese University of Hong Kong, Hong Kong, China

Simon Law Department of Surgery, The University of Hong Kong, Hong Kong, China

Derek Lee Division of Neurosurgery, Department of Surgery, Li Ka Shing Faculty of Medicine, The University of Hong Kong, Queen Mary Hospital, Hong Kong, China

Nikki P. Lee Department of Surgery, The University of Hong Kong, Hong Kong, China

Will M. Lee School of Biological Sciences, University of Hong Kong, Hong Kong, China

Gilberto K.K. Leung Division of Neurosurgery, Department of Surgery, Li Ka Shing Faculty of Medicine, The University of Hong Kong, Queen Mary Hospital, Hong Kong, China

Angela M. Liu Department of Pharmacology, National University of Singapore, Singapore

Department of Surgery, National University of Singapore, Singapore

Qian Liu Center of Medical Physics and Technology, Hefei Institute of Physical Science, Chinese Academy of Sciences, Hefei, China

John M. Luk Department of Pharmacology and Department of Surgery, National University of Singapore, Singapore

Department of Surgery, University of Hong Kong, Pokfulam, Hong Kong, China Institute of Molecular and Cell Biology, Biopolis, Singapore 
Jayakanthan Mannu Center for Bioinformatics, School of Life Sciences, Pondicherry University, Pondicherry, India

Premendu P. Mathur Center for Bioinformatics, School of Life Sciences, Pondicherry University, Pondicherry, India

KIIT University, Bhubaneshwar, Odisha, India

Dolores D. Mruk The Mary M. Wohlford Laboratory for Male Contraceptive Research, Center for Biomedical Research, Population Council, New York, NY, USA

Felix H. Shek Department of Surgery, The University of Hong Kong, Hong Kong, China

Han-Ming Shen Saw Swee Hock School of Public Health, Department of Physiology, Yong Loo Lin School of Medicine, National University of Singapore, Singapore

Bruno Silvestrini S.B.M. Srl, Rome, Italy

Rumani Singh National Institute of Advanced Industrial Science \& Technology (AIST), Tsukuba Science City, Japan

Stella Sun Division of Neurosurgery, Department of Surgery, Li Ka Shing Faculty of Medicine, The University of Hong Kong, Queen Mary Hospital, Hong Kong, China

Patrick Tan Cancer and Stem Cell Biology, Duke-NUS Graduate Medical School, Singapore

Yong Loo Lin School of Medicine, Cancer Science Institute of Singapore, National University of Singapore, Kent Ridge, Singapore

Genome Institute of Singapore, Singapore

Cellular and Molecular Research, National Cancer Centre of Singapore, Singapore

Renu Wadhwa National Institute of Advanced Industrial Science \& Technology (AIST), Tsukuba Science City, Japan

Wei Wang Department of Pharmacology, National University of Singapore, Singapore

Department of Surgery, National University of Singapore, Singapore, Singapore

Kwong-Fai Wong Cancer Science Institute of Singapore, National University of Singapore, Singapore

Weiliang Xia School of Biomedical Engineering and Med-X Research Institute, Shanghai Jiao Tong University, Shanghai, P.R. China

Xiang Xiao The Mary M. Wohlford Laboratory for Male Contraceptive Research, Center for Biomedical Research, Population Council, New York, NY, USA 
Nelson S. Yee Division of Hematology-Oncology, Department of Medicine, Penn State College of Medicine, Penn State Hershey Cancer Institute, Penn State Milton S. Hershey Medical Center, Pennsylvania State University, Hershey, PA, USA

Rosemary K. Yee Penn State Harrisburg School of Humanities, Pennsylvania State University, Middletown, PA, USA

Xia Zhao Department of Gynecology and Obstetrics, West China Second Hospital, Sichuan University, Chengdu, P.R. China

Shengtao Zhou Department of Gynecology and Obstetrics, West China Second Hospital, Sichuan University, Chengdu, P.R. China 